\title{
Spectral ranking
}

\author{
SEBASTIANO VIGNA \\ Dipartimento di informatica, Università degli Studi di Milano, Milano, 20122, Italy \\ (e-mail: sebastiano.vigna@unimi.it)
}

\begin{abstract}
We sketch the history of spectral ranking - a general umbrella name for techniques that apply the theory of linear maps (in particular, eigenvalues and eigenvectors) to matrices that do not represent geometric transformations, but rather some kind of relationship between entities. Albeit recently made famous by the ample press coverage of Google's PageRank algorithm, spectral ranking was devised more than 60 years ago, almost exactly in the same terms, and has been studied in psychology, social sciences, bibliometrics, economy, and choice theory. We describe the contribution given by previous scholars in precise and modern mathematical terms: Along the way, we show how to express in a general way damped rankings, such as Katz's index, as dominant eigenvectors of perturbed matrices, and then use results on the Drazin inverse to go back to the dominant eigenvectors by a limit process. The result suggests a regularized definition of spectral ranking that yields for a general matrix a unique vector depending on a boundary condition.
\end{abstract}

Keywords: ranking, centrality, eigenvectors

\section{Introduction}

From a mathematical viewpoint, a matrix $M$ represents a linear transformation between two linear spaces. It is just one of the possible representations of the mapit depends on a choice for the bases of the source and target space. Nonetheless, matrices arise all the time in many fields outside mathematics, often because they can be used to represent (weighted) binary relations. At that point, one can apply the full machinery of linear algebra and see what happens. The most famous example of this kind is probably spectral graph theory, which provides bounds for several graph features using eigenvalues of adjacency matrices.

When a square matrix represents relationships between entities, such as endorsement among persons, teams defeating other teams, friends or followers on social networks, and so on, several different eigenvectors can be obtained from the original matrix, giving rise to different kinds of spectral rankings. Spectral rankings have been computed under different names since the 50s in psychology, social sciences, bibliometrics, economy, and choice theory.

This paper describes the early history of spectral ranking, highlighting fundamental contributions. Moreover, by describing those contributions in modern terms, we can actually describe new connections between them and show that all rankings depending on a damping or attenuation factor, such as Katz's index, are just dominant eigenvectors of perturbed matrices. We then go back to the eigenvectors of the original matrix by a limiting process, obtaining a regularized definition of spectral 
ranking that yields for a general matrix a unique vector depending on a boundary condition.

\section{Spectral ranking 101}

Let us start with a square matrix $M$ on the reals. We will not make any assumption on $M$. We imagine that the indices of rows and columns actually correspond to some entity, and that each value $m_{i j}$ represents some form of endorsement or approval of entity $j$ from entity $i$. Endorsement can be negative, with the obvious meaning.

Many centrality indices based on simple summations performed on the row or columns of this matrix were common in psychometry and sociometry. For instance, if the matrix contains just zeroes and ones meaning "don't like" or "like," respectively, the sum of column $j$ will tell us how many entities like $j$. But, clearly, we are not making much progress.

The first fundamental step toward spectral ranking was made by Seeley (1949): He noted that these indices were not really meaningful because they did not take into consideration that it is important being liked by someone that is in turn being liked a lot, and so on. In other words, an index of importance, centrality, or authoritativeness, should be defined recursively ${ }^{1}$ so that my index is equal to the weighted sum of the indices of the entities that endorse me. In matrix notation, ${ }^{2}$

$$
r=r M
$$

Of course, this is not always possible. Seeley, however, considers a non-negative matrix without null rows and normalizes its rows so that they have unit $\ell_{1}$ norm (e.g., you divide each entry by its row sum); his rows have always non-zero entries, so this is always possible, and Equation (1) has a solution, because $M \mathbf{1}^{T}=\mathbf{1}^{T}$, so 1 is an eigenvalue of $M$, and its left eigenvector(s) provide solutions to Equation (1). Uniqueness is a more complicated issue which Seeley does not discuss and which can be easily analyzed using the well-known Perron-Frobenius theory of non-negative matrices, which also shows that 1 is the spectral radius, so $\boldsymbol{r}$ is a dominant ${ }^{3}$ eigenvector, and that there are positive solutions. ${ }^{4}$

Our discussion can be formally restated for right eigenvectors, but of course Seeley's motivation fails. However, Teh-Hsing Wei in his Ph.D. dissertation (1952) argues about ranking (sport) teams, reaching the following, dual conclusions. ${ }^{5}$ Given a matrix $M$ expressing how much team $i$ is better than team $j$ (e.g., 1 if $i$ beats $j$, $1 / 2$ for ties, 0 if $i$ loses against $j$, with coherent values in symmetric positions), Wei argues that an initial score ${ }^{6}$ of 1 given to all teams, leading to an ex aequo ranking,

1 The word "recursively," here, is used in a generalized but slightly incorrect sense which is common in the literature, and for which the word "impredicatively" would be more appropriate, but probably obscure to most readers.

2 We use row vectors.

3 A dominant eigenvalue is an eigenvalue with largest modulus (i.e., the spectral radius). An eigenvector associated with the dominant eigenvalue is called a dominant eigenvector. In most practical cases of spectral ranking, there is just one strictly dominant eigenvalue.

4 Actually, Seeley exposes the entire matter in terms of linear equations. Matrix calculus is used only for solving a linear system by Cramer's rule.

5 Kendall (1955) discusses Wei's (unpublished) findings at length.

6 Here, we take care of distinguishing the scores given to the teams from the ranking obtained sorting the teams by score. 
can be significantly improved as follows: Each team gets a new ranking obtained by adding the scores of the teams that it defeated, and half the scores of the team with whom there was a draw. There is thus a new set of scores and a new ranking, and so on. In other words, Wei suggests looking at the rank induced by the vector

$$
\lim _{k \rightarrow \infty} \frac{M^{k} \mathbf{1}^{T}}{\left\|M^{k} \mathbf{1}^{T}\right\|}
$$

Wei uses Perron-Frobenius theory to show that under suitable hypotheses this ranking stabilizes at some point to the one induced by the dominant right eigenvector. In modern terms, given a matrix $M$ expressing how much each team is better than another, the right dominant eigenvector provides the correct ranking of all teams. ${ }^{7}$

Wei's ranking is interesting in its own for three reasons: First, the motivation is clearly different; second, it clearly shows that using the dominant eigenvector (whatever the dominant eigenvalue) was already an established technique in the $50 \mathrm{~s}$; third, in this kind of ranking, the relevant convergence is in rank (the actual components of the vector are immaterial).

Few years later, in his celebrated book on graph theory Berge quotes Wei's thesis and makes the remark that the (right) eigenvector approach can be applied to any directed graph. As an example, he considers sociograms, where nodes represent individuals and arcs represent influence (Berge, 1958). ${ }^{8}$

Getting back to left eigenvectors, the works of Seeley, Wei, and Berge suggest that we consider matrices $M$ with a real and positive dominant eigenvalue $\lambda$ and its eigenvectors, that is, vectors $r$ such that

$$
\lambda \boldsymbol{r}=\boldsymbol{r} M
$$

If $\lambda$ is complex, $\boldsymbol{r}$ cannot be real, and the lack of an ordering that is compatible with the field structure makes complex numbers a bad candidate for ranking.

In general, a (left $)^{9}$ spectral ranking associated with $M$ is a dominant (left) eigenvector. If the eigenspace has dimension one, we can speak of the spectral ranking associated with $M$. Note that in principle such a ranking is defined up to a constant: This is not a problem if all coordinates of $\boldsymbol{r}$ have the same sign, but introduces an ambiguity otherwise.

\section{Damping}

We will now start from a completely different viewpoint. If the matrix $M$ is a zero/one matrix, the entry $i, j$ of $M^{k}$ contains the number of directed path from $i$ to $j$ in the directed graph defined by $M$ in the obvious way. A reasonable way of measuring the importance of $j$ could be measuring the number of paths going

7 This technique is known in the literature about ranking of sport teams as "Perron ranking" or "Kendall-Wei ranking."

8 Indeed, if we had to assign a name to the dominant eigenvector it should be "Wei-Berge index."

9 The distinction between left and right spectral ranking is in principle, of course, useless, as the left spectral ranking of $M$ is the right spectral ranking of $M^{T}$. Nonetheless, the kind of motivations leading to the two kind of rankings are quite different, and we feel that it is useful to keep around the distinction: If the matrix represents endorsement, left spectral ranking is the correct choice; if the matrix represents influence or "better-than" relationships, right spectral ranking should be used instead. 
into $j$, as they represent recursive endorsements. ${ }^{10}$ Unfortunately, trying the obvious, that is,

$$
\mathbf{1}\left(1+M+M^{2}+M^{3}+\cdots\right)=\mathbf{1} \sum_{k=0}^{\infty} M^{k}
$$

will not work, as formally the above equation is correct, but convergence is not guaranteed. Convergence is guaranteed, however, if $M$ has spectral radius smaller than one, that is, $\left|\lambda_{0}\right|<1$. It is thus tempting to introduce an attenuation or damping factor that makes things work:

$$
\mathbf{1}\left(1+\alpha M+\alpha^{2} M^{2}+\alpha^{3} M^{3}+\cdots\right)=\mathbf{1} \sum_{k=0}^{\infty}(\alpha M)^{k}
$$

Now, we are actually working with $\alpha M$, which has spectral radius smaller than one as long as $\alpha<1 /\left|\lambda_{0}\right|$ (e.g., if $M$ is (sub)stochastic any $\alpha<1$ will do the job). This index was proposed by Katz (1953). ${ }^{11}$ He notes that

$$
\mathbf{1} \sum_{k=0}^{\infty}(\alpha M)^{k}=\mathbf{1}(1-\alpha M)^{-1}
$$

which means that his index can be computed solving the linear system

$$
\boldsymbol{x}(1-\alpha M)=\mathbf{1}
$$

\section{Boundary conditions}

There is still an important ingredient we are missing: some initial preference, or boundary condition, as Hubbell (1965) calls it. Hubbell's interest is clique detection, an early study of spectral graph clustering. ${ }^{12}$ Hubbell is inspired by the works of Luce, Perry, and Festinger on clique identification (Luce \& Perry, 1949; Festinger, 1949); they use fixed powers of the adjacency matrix to estimate the similarity of nodes, and Hubbell proposes to sum up all powers of a matrix when such a sum exists. Then, in analogy with Leontief's input-output economic model, ${ }^{13}$ which represents the relationships between input and output of goods in each industry (Leontief,

10 Indeed, taking the limit of the vector giving for each node the number incoming paths of length $k$ (somehow normalized) when $k \rightarrow \infty$ leads to the definition of spectral ranking, as the process is equivalent to finding the dominant left eigenvector using the power method. This was observed already by Berge (1958).

11 We must note that actually Katz's index is $1 M \sum_{k=0}^{\infty}(\alpha M)^{k}$. This additional multiplication by $M$ is somewhat common in the literature; it is probably a case of horror vacui.

12 It would be interesting to write a note similar to this one for spectral clustering, as sociologists have been playing with the idea for quite a while.

13 Recently, Franceschet (2011) has argued that Leontief's input-output model is a precursor of PageRank, which would make it the oldest known example of spectral ranking. I think this is a red herring, as Leontief just wants to represent the relationship between input and output of an economy. He claims that an equilibrium is reached when prices are given by the fixpoint of the linear operator describing the input/output relationship, but being the goods indexing the matrix inhomogeneous, this pricing is not a ranking (and, indeed, Leontief does not appear to make claims in this direction). If we consider any study of fixpoints of a linear operator that expresses some kind of input/output relation a kind of spectral ranking, then Markov (1906) beats Leontief by more than 30 years, and we can probably go back further. 
1941), he argues that one can define a status index $r$ using the recursive equation

$$
\boldsymbol{r}=\boldsymbol{v}+\boldsymbol{r} M
$$

where $\boldsymbol{v}$ is a boundary condition, or exogenous contribution to the system. Finally, he notes that formally

$$
\boldsymbol{r}=\boldsymbol{v}(1-M)^{-1}=\boldsymbol{v} \sum_{k=0}^{\infty} M^{k}
$$

and that the right side converges as long as $\left|\lambda_{0}\right|<1: M$ can even have negative entries. Clearly, this is a generalization of Katz's index ${ }^{14}$ to general matrices that adds an initial condition, as the vector $\mathbf{1}$ is replaced by the more general boundary condition $\boldsymbol{v} .^{15,16}$

\section{From eigenvectors to path summation}

Seeley's, Wei's, and Katz's work might seem unrelated. Nothing could be farther from truth. Let's get back to the basic spectral ranking equation:

$$
\lambda_{0} r=r M
$$

When the eigenspace of $\lambda_{0}$ has dimension larger than one, there is no clear choice for $\boldsymbol{r}$. But we can try to perturb $M$ so that this happens. A simple way is using Brauer's results (1952) about eigenvector separation: ${ }^{17}$

\section{Theorem 1}

Let $A$ be an $n \times n$ complex matrix, $\lambda_{0}, \lambda_{1}, \ldots, \lambda_{n-1}$ be the eigenvalues of $A$, and let $\boldsymbol{x}$ be a non-zero complex vector such that $A \boldsymbol{x}^{T}=\lambda_{0} \boldsymbol{x}^{T}$. Then, for every complex vector $\boldsymbol{v}$, the eigenvalues of $A+\boldsymbol{x}^{T} \boldsymbol{v}$ are $\lambda_{0}+\boldsymbol{v} \boldsymbol{x}^{T}, \lambda_{1}, \ldots, \lambda_{n-1}$.

Brauer's theorem suggests to perform a rank-one convex perturbation of $M$ using a vector $\boldsymbol{v}$ satisfying $\boldsymbol{v} \boldsymbol{x}^{T}=\lambda_{0}$ by applying the theorem to $\alpha M$ and $(1-\alpha) \boldsymbol{x}^{T} \boldsymbol{v}$ :

$$
\lambda_{0} \boldsymbol{r}=\boldsymbol{r}\left(\alpha M+(1-\alpha) \boldsymbol{x}^{T} \boldsymbol{v}\right)
$$

Now, $\alpha M+(1-\alpha) \boldsymbol{x}^{T} \boldsymbol{v}$ has the same dominant eigenvalue of $M$, but with algebraic multiplicity one, and all other eigenvalues are multiplied by $\alpha$. This ensures that we have a unique $\boldsymbol{r}$, at the price of having introduced a parameter (the choice of $\boldsymbol{x}$ is particularly simple in case $M$ is stochastic, as in that case, we can take 1).

There is also another important consequence: $\boldsymbol{r}$ is defined up to a constant, so we can impose that $\boldsymbol{r} \boldsymbol{x}^{T}=\lambda_{0}$ (i.e., in case $\boldsymbol{x}=\mathbf{1}$, that the sum of $\boldsymbol{r}$ 's coordinates is $\lambda_{0}$, which implies, if all coordinates have the same sign, that $\|\boldsymbol{r}\|_{1}=\lambda_{0}$ ). We obtain

$$
\lambda_{0} \boldsymbol{r}=\alpha \boldsymbol{r} M+(1-\alpha) \lambda_{0} \boldsymbol{v}
$$

14 Hubbell claims that its index (actually, its status model) bears a "rough resemblance" to Katz's: Once the mathematics has been laid out in simple terms, one can easily see that they are the same thing.

15 We note that while the ranking induced by $1 M(1-\alpha M)^{-1}$ and $\mathbf{1}(1-\alpha M)^{-1}=\mathbf{1}+\mathbf{1} \alpha M(1-\alpha M)^{-1}$ is the same, this is no longer true when we use a general boundary condition.

16 Hubbell remarks that its score depends linearly on the boundary condition. This is actually an important feature for quick computation of personalized or topical versions of PageRank (Jeh \& Widom, 2003).

17 I learned the usefulness of Brauer's results in this context for separating eigenvalues from Stefano Serra-Capizzano. The series of papers by Brauer is also (maybe not surprisingly) quoted by Katz in his paper (Katz, 1953). 
so now

$$
\boldsymbol{r}=(1-\alpha) \boldsymbol{v}\left(1-\alpha M / \lambda_{0}\right)^{-1}=(1-\alpha) \boldsymbol{v} \sum_{k=0}^{\infty}\left(\alpha M / \lambda_{0}\right)^{k}=\left(1-\lambda_{0} \beta\right) \boldsymbol{v} \sum_{k=0}^{\infty}(\beta M)^{k}
$$

and the summation certainly converges if $\alpha<1$ (or, equivalently, if $\beta<1 / \lambda_{0}$ ). In other words, Katz-Hubbell's index (modulo a multiplicative constant) can be obtained as the spectral ranking of a rank-one perturbation of the original matrix. ${ }^{18}$ As the convex perturbation parameter $\alpha$ moves from 0 to 1 , the damping factor $\beta$ moves from 0 to $1 / \lambda_{0}$.

\section{From path summation to eigenvectors}

A subtler reason takes us backwards. Given a matrix $Z$, the index of $Z$ is the smallest non-negative integer $k$ such that the kernel (the eigenspace associated to the eigenvalue zero) of $Z^{k}$ is equal to the kernel of $Z^{k+1}$. Equivalently, the index is the size of the largest Jordan block of the eigenvalue zero (which is zero for a non-singular matrix).

The Drazin inverse of $Z$, written $Z^{D}$, is the unique solution of the equations

$$
Z X Z=X \quad X Z=Z X \quad Z^{v+1} X=Z^{v},
$$

where $v$ is the index of $Z$ (Drazin, 1958). When $Z$ is non-singular, it coincides with $Z^{-1}$. The matrix $\llbracket Z \rrbracket=1-Z Z^{D}$ is called the eigenprojection (for the eigenvalue zero) of $Z$ : It is a projection on the kernel of $Z^{v}$ along the range of $Z^{v}$. The fundamental theorem we will use about the Drazin inverse is due to Meyer (1974):

\section{Theorem 2}

Let $Z$ be a square matrix with index $v$. If $m$ and $p$ are non-negative integers, the limit

$$
\lim _{\varepsilon \rightarrow 0} \varepsilon^{m}(\varepsilon+Z)^{-1} Z^{p}
$$

exists if and only if $m+p \geqslant v$, and in that case

$$
\lim _{\varepsilon \rightarrow 0} \varepsilon^{m}(\varepsilon+Z)^{-1} Z^{p}= \begin{cases}Z^{D} Z^{p} & \text { if } m=0 \\ (-1)^{m-1} Z^{m+p-1} \llbracket Z \rrbracket & \text { if } m>0\end{cases}
$$

We would like to know what happens to the perturbed dominant eigenvector (5) when $\alpha \rightarrow 1$ :

$$
\begin{aligned}
\lim _{\alpha \rightarrow 1}(1-\alpha) \boldsymbol{v}\left(1-\alpha M / \lambda_{0}\right)^{-1} & =\lim _{\alpha \rightarrow 1} \frac{1-\alpha}{\alpha} \boldsymbol{v}\left(\frac{1}{\alpha}-M / \lambda_{0}\right)^{-1} \\
& =\lim _{\alpha \rightarrow 1} \frac{1-\alpha}{\alpha} \boldsymbol{v}\left(\frac{1}{\alpha}-1+1-M / \lambda_{0}\right)^{-1} \\
& =\lim _{\alpha \rightarrow 1} \frac{1-\alpha}{\alpha} \boldsymbol{v}\left(\frac{1-\alpha}{\alpha}+\left(1-M / \lambda_{0}\right)\right)^{-1} \\
& =\lim _{\varepsilon \rightarrow 0} \varepsilon \boldsymbol{v}\left(\varepsilon+\left(1-M / \lambda_{0}\right)\right)^{-1}
\end{aligned}
$$

18 This result is of course well known for stochastic matrices, as it is one of the ways of defining PageRank. 
Now, $1-M / \lambda_{0}$ is a singular matrix with index equal to the index ${ }^{19}$ of $\lambda_{0}$ in $M$. If $\lambda_{0}$ is semisimple (i.e., $v=1$ ), we can apply Meyer's theorem with $m=1, p=0$ and conclude that

$$
\lim _{\alpha \rightarrow 1}(1-\alpha) \boldsymbol{v}\left(1-\alpha M / \lambda_{0}\right)^{-1}=\boldsymbol{v} \llbracket 1-M / \lambda_{0} \rrbracket
$$

The circle is closed, as the kernel of $1-M / \lambda_{0}$ is exactly the eigenspace of $M$ associated with the eigenvalue $\lambda_{0}$ : Spectral ranking is just the limit case of KatzHubbell's index. ${ }^{20}$

The eigenprojection $\llbracket 1-M / \lambda_{0} \rrbracket$ has an intuitive description if $\lambda_{0}$ and all other eigenvalues of maximum modulus are semisimple: It is equal (Rothblum, 1981) to the Cesàro limit

$$
\lim _{n \rightarrow \infty} \sum_{k=0}^{n-1} \frac{\left(M / \lambda_{0}\right)^{k}}{n}
$$

that is, the limit in average of $\left(M / \lambda_{0}\right)^{n}$. In stochastic matrices, all eigenvalues of modulo one are semisimple, which explains why the Cesàro limit is very popular in the Markov chain literature to obtain eigenprojections. Rothblum (1981) shows that a suitable generalization of the Cesàro limit to n-fold sums makes it possible to write the eigenprojections in limit form even if the eigenvalues of maximum modulus other than $\lambda_{0}$ are not semisimple.

If $\lambda_{0}$ is not semisimple, say with index $v$, the situation is not very different: Meyer's theorem essentially tells us that the perturbed dominant eigenvector (5) would grow too quickly as $\alpha \rightarrow 1$, but nonetheless

$$
\lim _{\alpha \rightarrow 1}(1-\alpha)^{v} \boldsymbol{v}\left(1-\alpha M / \lambda_{0}\right)^{-1}=(-1)^{v-1} \boldsymbol{v} \llbracket 1-M / \lambda_{0} \rrbracket\left(1-M / \lambda_{0}\right)^{v-1}
$$

The resulting vector is still in the kernel of $1-M / \lambda_{0}$, because $\llbracket 1-M / \lambda_{0} \rrbracket$ projects on the kernel of $\left(1-M / \lambda_{0}\right)^{v}$ :

$$
\left(\boldsymbol{v} \llbracket 1-M / \lambda_{0} \rrbracket\left(1-M / \lambda_{0}\right)^{v-1}\right)\left(1-M / \lambda_{0}\right)=\boldsymbol{v} \llbracket 1-M / \lambda_{0} \rrbracket\left(1-M / \lambda_{0}\right)^{v}=0
$$

Note that since the $(1-\alpha)^{v}$ factor is just a scalar, we can also write (even when $v=1)$

$$
\lim _{\alpha \rightarrow 1} \frac{\boldsymbol{v}\left(1-\alpha M / \lambda_{0}\right)^{-1}}{\left\|\boldsymbol{v}\left(1-\alpha M / \lambda_{0}\right)^{-1}\right\|}=\frac{(-1)^{v-1} \boldsymbol{v} \llbracket 1-M / \lambda_{0} \rrbracket\left(1-M / \lambda_{0}\right)^{v-1}}{\left\|\boldsymbol{v} \llbracket 1-M / \lambda_{0} \rrbracket\left(1-M / \lambda_{0}\right)^{v-1}\right\|}
$$

which means that the direction of the perturbed dominant eigenvector always tends to that of a dominant eigenvector of $M$ as $\alpha \rightarrow 1$, albeit normalization is necessary to avoid divergence. If $M$ is non-negative, Rothblum shows that generalized Cesàro limits can be applied even to this case.

\section{Putting it all together}

It is interesting to note that the journey made by our original definition through perturbation and then limiting has an independent interest. We started with a matrix

19 The index of the eigenvalue $\lambda$ of a matrix $M$ is the index of the matrix $1-M / \lambda$, or equivalently the maximal dimension of the Jordan blocks of $M$ containing $\lambda$.

20 Once again, this result is well known for PageRank (Boldi et al., 2009). 
$M$ with possibly many eigenvectors associated with the dominant eigenvalue, and we ended up with a specific eigenvector associated with $\lambda_{0}$, given the boundary condition $\boldsymbol{v}$. This suggests to define in general the spectral ranking ${ }^{21}$ of $M$ with boundary condition $\boldsymbol{v}$ when the dominant eigenvalue $\lambda_{0}$ is semisimple as

$$
\boldsymbol{r}=\boldsymbol{v} \llbracket 1-M / \lambda_{0} \rrbracket
$$

or, in the general case,

$$
\boldsymbol{r}=\boldsymbol{v}(-1)^{v-1} \llbracket 1-M / \lambda_{0} \rrbracket\left(1-M / \lambda_{0}\right)^{v-1}
$$

with $v$ equal to the index of $\lambda_{0}$. If $M$ has a unique eigenvector, this definition is equivalent to Equation (2), and $\boldsymbol{v}$ is immaterial. However, in the general case (6) provides a unique (albeit possibly difficult to compute) eigenvector depending on $\boldsymbol{v}$.

If we start from a generic non-negative matrix $M$ and assume to normalize its rows, obtaining a substochastic matrix $P$, we should probably speak of Markovian spectral ranking, as the Markovian nature of the object becomes dominant. If $\lambda_{0}=1$, we have

$$
\boldsymbol{r}=\boldsymbol{v} \llbracket 1-P \rrbracket=\boldsymbol{v} \lim _{n \rightarrow \infty} \sum_{k=0}^{n-1} \frac{P^{k}}{n}
$$

as dictated by Markov chain theory. ${ }^{22}$ If $\boldsymbol{v}$ is a distribution, $\boldsymbol{r}$ is essentially ${ }^{23}$ the limit distribution when the chain is started with distribution $\boldsymbol{v}$. If $\lambda_{0}<1$, we must check its index and work the details as in the general case (6), as we can no longer guarantee semisemplicity of all eigenvalues of maximum modulus. For example, the matrix

$$
\left(\begin{array}{ccc}
0 & \frac{1}{2} & \frac{1}{2} \\
0 & 0 & 1 \\
0 & 0 & 0
\end{array}\right)
$$

has a single eigenvalue 0 with algebraic multiplicity three and geometric multiplicity one.

Finally, we could define the damped spectral ranking of $M$ with boundary condition $\boldsymbol{v}$ and damping factor $\alpha$ when the dominant eigenvalue $\lambda_{0}$ of $M$ is semisimple as

$$
\boldsymbol{r}_{\alpha}=(1-\alpha) \boldsymbol{v} \sum_{k=0}^{\infty}\left(\alpha M / \lambda_{0}\right)^{k}
$$

for $|\alpha|<1$. If we normalize the rows of $M$, we should speak of a damped Markovian spectral ranking (see Table 1 ).

The $(1-\alpha)$ term comes out naturally from Equation (5), and makes the limit for $\alpha \rightarrow 1$ exist in the semisimple case, unifying the treatment of normalized (i.e., Markovian) and unnormalized rankings; moreover, it forces $\|\boldsymbol{r}\|_{1}=1$ when $M$ is stochastic and $\boldsymbol{v}$ is a distribution. ${ }^{24}$ An even more general definition, taking the index

21 We remark that in social sciences and social-network analysis "eigenvector centrality" is often used to name collectively ranking techniques using eigenvectors ("centrality" is the sociologist's "ranking"). On the other hand, in those areas indices based on paths such as Katz's are considered to be different beasts.

22 Substochastic matrices, too, enjoy the property that all eigenvalues of modulo one, if any, are semisimple; however, if $\lambda_{0}<1$, there are no such eigenvalues.

23 "Essentially" because $\llbracket 1-P \rrbracket$ smooths out problems due to periodicities in the matrix.

24 As noted by Bonacich (1987), $\alpha$ can even be negative. 
Table 1. A reasoned table of the basic types of spectral ranking; the names defined in Section 7 are in boldface.

\begin{tabular}{lcc}
\hline \hline & No normalization & Row normalization \\
\hline \multirow{3}{*}{ No damping } & Spectral ranking & Markovian spectral ranking \\
& Eigenvector centrality & Markov chain steady state \\
& (Wei, 1952; Berge, 1958) & (Seeley, 1949) \\
& Damped spectral ranking & Damped Markovian spectral ranking \\
Damping & Katz's index & Total effect centrality, PageRank \\
& (Katz, 1953) & (Friedkin, 1991; Page et al., 1998) \\
\hline \hline
\end{tabular}

$v$ of $\lambda_{0}$ into consideration, would use a multiplicative factor $(1-\alpha)^{v}$, which would make the limit for $\alpha \rightarrow 1$ always exist. It can be argued, however, that for practical purposes the normalization factor is just a nuisance, as it does not affect ranks (just scores), and it might be difficult to determine, as it depends on the index of $\lambda_{0}$.

It is interesting to note that in the Markovian case the change of role of the boundary condition from the damped to the non-damped case has a simple interpretation: In the damped case, we have a Markov chain that restarts on a fixed distribution $\boldsymbol{v}$, and there is a single stationary distribution which is the limit of every starting distribution; in the non-damped case, $\boldsymbol{v}$ is the starting distribution from which we compute the limit distribution using the eigenprojection. Thus, when $\alpha \rightarrow 1$, the restart distribution $\boldsymbol{v}$ becomes the starting distribution, which is significant only if the graph underlying the chain has more than one terminal strongly connected component. An analogous consideration can be done in the general semisimple case: The preference vector of Katz-Hubbell's index becomes, in the limit, the starting vector of an averaged version of the power method that mimics Cesàro limit.

\section{Followers}

The work of Seeley was almost unnoticed, Wei's dissertation was known mainly to rank theorists, Berge's observations were buried in a book on graph theory, and Katz's paper was known mainly by sociologists, so it is no surprise that spectral ranking has been rediscovered several times.

In this section, we gather the main insurgencies of spectral ranking in various fields we are aware of. In some cases, spectral ranking in some form is applied to some domain; in other cases, very mild variations of previous ideas are proposed (mostly, we must unhappily say, without motivation or assessment). The list is certainly incomplete.

(French Jr., 1956). For completeness, we mention French's theory of social power, which bears a superficial formal resemblance with spectral ranking. However, French's theory considers right eigenvectors of row-stochastic matrices, so the trivial uniform solution is always a solution, and it is considered a good solution, as the theory studies the formation of consensus (e.g., the probability of getting the trivial uniform solution depending on the structure of the graph). 
(Gould, 1967). Gould proposes to use eigenvectors to study geographical features such as irregular terrains, the orientation of transportation networks, and even the homogeneity of architectural features. In one particular example, he consider the connectivity graph on a set of cities (edges are direct roads) and discusses the information conveyed by the dominant and a few other eigenvectors (Seeley's, Wei's, and Berge's work are not quoted).

(Bonacich, 1972). Bonacich proposes to use spectral ranking on zero-one matrices representing entities and their relationships to identify the most important entities (Seeley's, Wei's, and Berge's work are not quoted).

(Pinski \& Narin, 1976). Here, $M$ is the matrix that contains in position $m_{i j}$ the number of references from journal $j$ to journal $i$. The matrix is then normalized in a slightly bizarre way, that is, by dividing $m_{i j}$ by the $j$ th [sic] row sum. The spectral ranking on this matrix is then used to rank journals. Geller (1978) tries to bring Markov-chain theory in by suggesting to divide by the $i$ th row sum instead (i.e., Markovian spectral ranking). No reference is made to previous work.

(Hoede, 1978). Hoede proposes to avoid the boundary condition of Hubbell's index by computing $1 M(1-M)^{-1}$ instead, under the condition that $1-M$ is invertible. This is exactly Katz's index with no damping. The main point of the author is that now we can just tweak the entries of $M$ so to make $1-M$ invertible, as "this hardly influences the model" [sic].

(Saaty, 1980). In the 70s, Saaty developed the theory of the analytic hierarchy process, a structured technique for dealing with complex decision. After some preprocessing, a table comparing a set of alternatives pairwise is filled with "better then" values (the entry $m_{i j}$ means how much $i$ is better than $j$, and the matrix must be reciprocal, i.e., $\left.m_{i j}=1 / m_{j i}\right)$; right spectral ranking is then used to rank the alternatives. Some insight as to why this is sensible can be found in Saaty (1987). The mathematics is of course identical to Wei's and Berge's, as the motivation is structurally similar.

(Bonacich, 1987). Bonacich proposes a mild extension of Katz's index (i.e., damped spectral ranking) that includes negative damping; the interpretation proposed is that in bargaining having a powerful neighbor should count negatively.

(Bonacich, 1991). Bonacich proposes to rank individuals and groups simultaneously, given the person/group incidence matrix, by computing the first left and right singular vectors of the incidence matrix $M$, which are the spectral rankings of $M M^{T}$ and $M^{T} M$, respectively.

(Friedkin, 1991). Friedkin discusses a damped version of French's consensus theory based on a row-stochastic matrix $M$. However, halfway through the paper he defines the total effect centrality of an entity as the average of the off-diagonal entries of a column of $(1-\alpha)(1-\alpha M)^{-1}$. If we forget the motivations behind Friedkin's model and take the average of all entries, instead, we obtain a damped (Markovian) spectral ranking of $M$ with constant boundary condition. If $M$ is obtained by normalizing 
the rows of the adjacency matrix of a graph, we obtain exactly PageRank (Page et al., 1998).

(Keener, 1993). Keener discusses a few different rankings for football teams based on the results of played games. He computes dominant eigenvectors of different matrices derived from game outcomes. This paper is one of the very few ones explicitly quoting both Wei and Berge.

(Page et al., 1998). PageRank is the damped Markovian spectral ranking of the adjacency matrix of a web graph (i.e., the graph having pages as nodes and hypertextual links as arcs). It was one the features originally used by Google to rank documents ${ }^{25}$ associated with a query, and it has since then been applied to a wide variety of problems (Gleich, 2015). The boundary condition is called preference vector, and it can be used to bias PageRank with respect to a topic or to personal preferences. In case the preference vector is constant, the resulting centrality is almost identical to the total effect centrality defined by Friedkin (1991), whose work is not quoted, though.

(Huberman et al., 1998). With the aim of predicting the number of visits to a web page, Huberman, Pirolli, Pitkow, and Lukose study a model derived from spreading activation networks. Essentially, given a distribution $d$ that tells which fraction of surfers are still surfing after time $t$, the prediction vector at time $t$ is $d(t) \boldsymbol{v} P^{t}$, where $\boldsymbol{v}$ is the initial number of surfers at each page. They use an inverse Gaussian distribution obtained experimentally, but using a geometric distribution the predicted overall (i.e., summed up over all $t$ ) number of surfers at each page will give a Markovian damped spectral ranking.

(Kleinberg, 1999). HITS is Kleinberg's algorithm for finding authorities and hubs in a (part of a) web graph. As in Bonacich's previous work (Bonacich, 1991), HITS computes the first left and right singular vectors of a matrix $A$, which are the spectral ranking of $A A^{T}$ and $A^{T} A$, respectively. Kleinberg notes, however, that it is possible to extract clustering information from additional singular vectors.

(Bonacich \& Lloyd, 2001). Bonacich and Lloyd propose again to use damped spectral ranking, but with a boundary condition, that is, Katz-Hubbell's index. The authors do quote Hubbell (1965), but apparently they do not realize that they are just redefining his index. They prove, however, prove that under strong conditions ( $M$ symmetric and with a strictly dominant eigenvalue) damped spectral ranking converges to spectral ranking.

25 It should be noted there are several other ways to use a graph to obtain scores for documents. For instance, one can use links (in particular, hypertext links) to alter text-based scores using the score of pointed pages: This simple idea dates at least back to the end of the 80s (Frisse, 1988). In the 90s, the idea was rediscovered again for the web: see, for example, Marchiori (1997), which, in spite of some claims floating around the net, does not do any kind of spectral ranking. An obvious spectral approach would use a preference vector containing normalized text-based scores, and then a right or left spectral ranking depending on whether authoritativeness or relevance is to be scored. To the knowledge of the author, this approach has not been explored yet. 
(Bergstrom et al., 2008). Eigenfactor is a score computed to score journals. It is a Markovian damped spectral ranking computed on the citation matrix, with an additional non-damped step (e.g., $S(1-\alpha S)^{-1}$ ), as in the original formulation of Katz's index.

\section{Conclusions}

We described a comprehensive framework for spectral ranking, highlighting the fundamental contributions of Seeley, Wei, and Berge (the dominant eigenvector, possibly with stochastic normalisation), Katz (damping), and Hubbell (boundary condition). We showed in a very general setting that one can move from dominant eigenvectors to damped rankings by a perturbation process, and get back to the dominant eigenvectors by taking the limit of the parameter controlling the perturbation. The process suggests a generic way to define spectral ranking by dominant eigenvectors on general graphs, by applying the eigenprojector of the (possibly normalized) adjacency matrix to a given boundary condition.

\section{Acknowledgments}

The author would like to thank David Gleich for many useful discussions and comments.

\section{References}

Berge, C. (1958). Théorie des graphes et ses applications. Paris, France: Dunod.

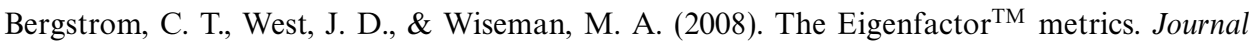
of Neuroscience, 28(45), 11433-11434.

Boldi, P., Santini, M., \& Vigna, S. (2009). PageRank: Functional dependencies. ACM Transactions on Information Systems, 27(4), 1-23.

Bonacich, P. (1972). Factoring and weighting approaches to status scores and clique identification. Journal of Mathematical Sociology, 2(1), 113-120.

Bonacich, P. (1987). Power and centrality: A family of measures. The American Journal of Sociology, 92(5), 1170-1182.

Bonacich, P. (1991). Simultaneous group and individual centralities. Social Networks, 13(2), 155-168.

Bonacich, P., \& Lloyd, P. (2001). Eigenvector-like measures of centrality for asymmetric relations. Social Networks, 23(3), 191-201.

Brauer, A. (1952). Limits for the characteristic roots of a matrix. IV: Applications to stochastic matrices. Duke Mathematical Journal, 19(1), 75-91.

Drazin, M. P. (1958). Pseudo-inverses in associative rings and semigroups. The American Mathematical Monthly, 65(7), 506-514.

Festinger, L. (1949). The analysis of sociograms using matrix algebra. Human Relations, 2(2), 153-9.

Franceschet, M. (2011). PageRank: Standing on the shoulders of giants. Communications of the $A C M, \mathbf{5 4}(6), 92-101$.

French Jr., J. R. P. (1956). A formal theory of social power. Psychological Review, 63(3), 181-194.

Friedkin, N. E. (1991). Theoretical foundations for centrality measures. The American Journal of Sociology, 96(6), 1478-1504. 
Frisse, M. E. (1988). Searching for information in a hypertext medical handbook. Communications of the ACM, 31(7), 880-886.

Geller, N. L. (1978). On the citation influence methodology of Pinski and Narin. Information Processing \& Management, 14(2), 93-95.

Gleich, D. F. (2015). PageRank beyond the web. SIAM Review, 57(3), 321-363.

Gould, P. R. (1967). On the geographical interpretation of eigenvalues. Transactions of the Institute of British Geographers, 42, 53-86.

Hoede, C. (1978). A new status score for actors in a social network. Memorandum 243. Twente University Department of Applied Mathematics.

Hubbell, C. H. (1965). An input-output approach to clique identification. Sociometry, 28(4), 377-399.

Huberman, B. A., Pirolli, P. L.T., Pitkow, J. E., \& Lukose, R. M. (1998). Strong regularities in world wide web surfing. Science, $\mathbf{2 8 0}(5360), 95$.

Jeh, G., \& Widom, J. (2003). Scaling personalized web search. In Proceedings of the 12th International World Wide Web Conference. New York, NY: ACM Press.

Katz, L. (1953). A new status index derived from sociometric analysis. Psychometrika, 18(1), 39-43.

Keener, J. P. (1993). The Perron-Frobenius theorem and the ranking of football teams. SIAM Review, 35(1), 80-93.

Kendall, M. G. (1955). Further contributions to the theory of paired comparisons. Biometrics, 11(1), 43-62.

Kleinberg, J. M. (1999). Authoritative sources in a hyperlinked environment. Journal of the $A C M$, 46(5), 604-632.

Leontief, W. W. (1941). The structure of American economy, 1919-1929: An empirical application of equilibrium analysis. Cambridge, MA: Harvard University Press.

Luce, R. D., \& Perry, A. D. (1949). A method of matrix analysis of group structure. Psychometrika, 14(2), 95-116.

Marchiori, M. (1997). The quest for correct information on the Web: Hyper search engines. Computer Networks and ISDN Systems, 29(8), 1225-1235.

Markov, A. A. (1906). Rasprostranenie zakona bolshih chisel na velichiny, zavisyaschie drug ot druga. Izvestiya Fiziko-matematicheskogo obschestva pri Kazanskom universitete, 2(15), $135-156$.

Meyer, Jr., C. D. (1974). Limits and the index of a square matrix. SIAM Journal on Applied Mathematics, 26(3), 469-478.

Page, L., Brin, S., Motwani, R., \& Winograd, T. (1998). The PageRank citation ranking: Bringing order to the web. Tech. rept. SIDL-WP-1999-0120. Stanford Digital Library Technologies Project, Stanford University.

Pinski, G., \& Narin, F. (1976). Citation influence for journal aggregates of scientific publications: Theory, with application to the literature of physics. Information Processing \& Management, 12(5), 297-312.

Rothblum, U. G. (1981). Expansions of sums of matrix powers. SIAM Review, 23(2), 143-164.

Saaty, T. L. (1980). The analytical hierarchy process. New York: McGraw-Hill.

Saaty, T. L. (1987). Rank according to Perron: A new insight. Mathematics Magazine, 60(4), 211-213.

Seeley, J. R. (1949). The net of reciprocal influence: A problem in treating sociometric data. Canadian Journal of Psychology, 3(4), 234-240.

Wei, T.-H. (1952). The algebraic foundations of ranking theory. Ph.D. thesis, University of Cambridge. 